\title{
ESTUDIO DE LA VARIACIÓN DEL PENSAMIENTO DIVERGENTE EN FÍSICA SEGÚN LA EDAD DE LOS ALUMNOS
}

\author{
PASTOR SARRO, SEGUNDO y PÉREZ FERNÁNDEZ, PEDRO \\ Grupo de Trabajo de Didáctica de las Ciencias Experimentales, CSIC. Vitrubio, 8. 28006 Madrid
}

\begin{abstract}
SUMMARY
Divergent Thinking, DT, is a mental mechanism which takes part in the process of solving problems having more than one valid solution. It is considered as a fundamental aspect of creativity. In our research about DT in Physics, we intended to present a new method, using the Guilford's E.I. tridimensional model as a referential framework, since we are convinced that it is crucially important to have an educational system which emphasizes creativity and the skills of the future scientist so that he/she will be able to face any challenging situation. In this paper we study the DT variation with age in Physics, and we have found out that younger students are likely to show better progress.
\end{abstract}

\section{INTRODUCCIÓN}

El trabajo que exponemos en el presente artículo forma parte de una línea de investigación que nuestro grupo de trabajo viene realizando en el campo de la didáctica de las ciencias experimentales en aspectos relacionados con las interacciones entre la creatividad, en concreto el pensamiento divergente (PD), y otras variables que, a nuestro parecer, son fundamentales en física; todo ello tomando como referencia el modelo EI de la estructura del intelecto de Guilford, en el que nuestras pruebas de $\mathrm{PD}$ en física tienen coordenadas concretas.

Ya que en trabajos anteriores hallamos indicios de que los alumnos de menor edad (nivel de $2^{\circ}$ de BUP) eran más susceptibles de experimentar cambios en dicha variable, en el presente trabajo nuestro objetivo fundamental ha sido comparar la variación del PD en física según la edad de los alumnos. Para ello dispusimos de dos grupos de $2^{\circ}$ de BUP (15 años) y dos de COU (17 años), que recibieron una enseñanza tradicional en física.

\section{ANTECEDENTES}

Es conveniente recordar que, así como la investigación científica ha tenido un enorme desarrollo en lo que va de siglo, la investigación en el campo de su didáctica no ha experimentado un desarrollo paralelo; hay un desfase entre la física tal como la concibe el científico y la física en el aula. De hecho, aún no hay en dicho campo una estructura elaborada; hay conocimientos y hay teorías, pero están dispersos, no organizados. Nosotros intentamos que exista algún día dicha estructura. Actualmente no hay textos sobre didáctica de la física, ni forma parte del currículo normal de las universidades. Hay que tener en cuenta que las variables en este ámbito no son tan concretas como en aquél, pues, aparte de su naturaleza, las muestras no son tan susceptibles de repetitividad, ya que no son recuperables al evolucionar los alumnos, al no ser los mismos de un curso a otro, etc. Aquí no se dan las condiciones de precisión ni la repetitividad habituales en la investigación científica. 
A partir de la obra Hereditary Genius (Galton, 1869), los investigadores han intentado descubrir la naturaleza de la creatividad. Galton llevó a cabo diversas investigaciones científicas sobre la creatividad científica y el genio. Así, por ejemplo, estableció que la creatividad no se trataba de algo accidental, sino de un fenómeno natural susceptible de estudiarse científicamente.

No necesitamos remontarnos a más de cuarenta años para encontrarnos con investigaciones sistemáticas sobre la creatividad en el campo de la ciencia. Citemos como ejemplo las realizadas por Stein en la Universidad de Utah: trabajó sobre exámenes biográficos de 1.600 científicos que fueron encuestados y concluyó algo que no ha sido refutado, a saber, que la creatividad científica puede desarrollarse por procedimientos determinados.

Actualmente, el pensamiento o producción divergente (mecanismo mental que interviene en la resolución de problemas que admiten varias soluciones, todas ellas válidas), si bien no es identificado totalmente con la creatividad, sí es considerado un componente fundamental. El estudio científico de la producción divergente (PD) tiene su origen en los trabajos de Guilford (1967) concretados en su modelo tridimensional (EI) de la estructura del intelecto. Este modelo nos sirve como sistema de referencia, ya que de los diversos modelos propuestos para el intelecto desde distintos campos de investigación es éste el que, a nuestro juicio, ofrece amplias posibilidades en el campo de la metodología de la enseñanza de la ciencia, tanto en la investigación como en la práctica docente.

Básicamente es un modelo tridimensional, que contiene tres de dichas dimensiones (Fig.1):

Contenidos: Discriminaciones básicas de la mente.

Operaciones: Procesos mentales o intelectuales.

Producto: Resultados de dichos procesos.

Como en el modelo, se consideran 5 operaciones, 6 productos y 4 contenidos; el cubo se divide en 120 celdillas, correspondiendo cada una a una capacidad o modalidad intelectiva determinada.

De dichas capacidades o actividades mentales, nosotros hemos elegido las 24 correspondientes a actividades mentales divergentes (oscuro en figura 1) y, dentro de éstas, a las de contenido semántico (referentes al significado de las cosas o los hechos), ya que lo consideramos fundamental en la comprensión de los fenómenos físicos. Así, para nuestro trabajo, hemos elaborado unas pruebas aplicadas a la física (Pérez y Civantos, 1985), que mostramos en el anexo y que corresponden a las celdillas o actividades mentales representadas en la figura 1 .

Así, por ejemplo, cuando se pide a un alumno que cite los posibles usos de una jeringa en mecánica de fluidos, los parámetros adecuados serían:
Figura 1

Cubo de Guilford.

Producción divergente de unidades semánticas, DMU. Producción divergente de clases semánticas, DMC.

Producción divergente de relaciones semánticas, DMR.

Producción divergente de sistemas semánticos, DMS

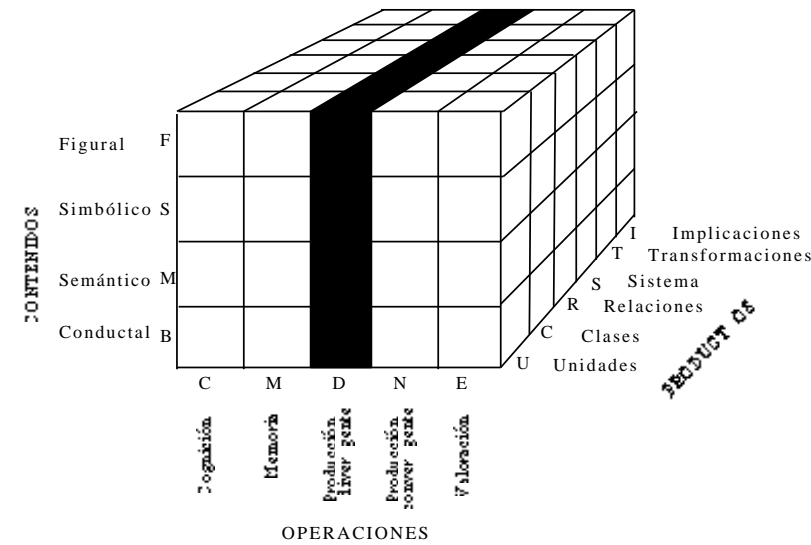

Operación: producción divergente (D), contenido: semántico (M), producto: unidad (U).

Por tanto, la designación correspondiente sería DMU.

También se determinan, según la prueba, las variables «fluencia mental» $(\mathrm{F})$ o número de respuestas válidas, «flexibilidad» (fx) o número de veces que el sujeto cambia el tipo de respuesta, «originalidad» $(\mathrm{O})$ o calificación a un comportamiento o respuesta por su carácter novedoso, no habitual, pero correcto físicamente, y «elaboración» (E) o número de elementos que entran en cada respuesta.

Al no haber encontrado trabajos relacionados con la PD en física que usen las capacidades semánticas según el modelo de Guilford, no hemos hallado investigaciones sobre fluencia, flexibilidad, originalidad y elaboración según dicho modelo y en dicha materia.

Los trabajos de investigación que conocemos sobre creatividad y PD en física y en su didáctica han venido basándose generalmente en dos vías diferentes: en cuestionarios realizados a científicos supuestamente creativos o en la adaptación de tests generales sobre creatividad como el «Torrance Parallel Lines Ability» (TPLA) o el «Torrance Product Improvement Activity» (TPIA) que fueron adaptados por Abramson del test de Torrance (Rieben, 1978). La metodología que nosotros aportamos utilizando como referencia el modelo EI de Guilford nos parece más operativa, de mejor cuantificación y con menos incidencia de los conocimientos previos.

Con ello intentamos aportar una nueva metodología en la investigación sobre la producción divergente y la crea- 
tividad en física, usando dicho modelo. Ello supone una nueva forma de evaluar la creatividad científica, aplicable al proceso de enseñanza-aprendizaje de la física más adecuado, a nuestro parecer, que el «Torrance Test of Creative Thinking».

Entre los aspectos que estamos estudiando, el presente trabajo trata la variación del PD en física según la edad y forma parte de la tesis doctoral presentada en la Facultad de Ciencias de la Universidad Autónoma de Madrid el 26 de noviembre de 1993 (Pastor, 1993).

Los trabajos que encontramos se refieren sobre todo a estudios en sujetos ya maduros en los que se investiga el posible declive de la creatividad general (Jennifer, 1993) o la creatividad en los científicos (Tsang, 1974) y, aunque la idea dominante es que la creatividad experimenta un declive con la edad (Abra, 1989), determinadas investigacions aportan, cuando menos, importantes matizaciones en el sentido de que el declive fisiológico no va necesariamente paralelo al de aspectos psicológicos como pueda ser la creatividad y que lo que pudiera interpretarse como un declive puede deberse a que el pensamiento creativo es diferente en distintas etapas de la vida al producirse cambios en los mecanismos creativos básicos (Jennifer,1993). Asimismo, aspectos como la experiencia juegan un papel en los cambios de la naturaleza de la creatividad (Sternberg, 1990).

En cuanto a la creatividad científica, si bien algunas investigaciones parecen mostrar un declive de la creatividad a partir de los 36-40 años (Owens, 1973), según otras posteriores, dicho declive se retrasaría apreciablemente (Tsang, 1974).

Nosotros estamos más interesados en edades en las que el sujeto se halla en proceso de formación (en nuestro caso alumnos de BUP y COU) cuando los cambios que pudieran esperarse fueran más notables.

En este aspecto hemos tratado de continuar otro trabajo de nuestro grupo (Pérez, Civantos y Gastesi, 1987) que incluyó alumnos de $2^{\circ}$ de BUP (un grupo $2 \mathrm{~A}$ de control y otro $2 \mathrm{~B}$ que recibió una enseñanza divergente), estudiantes de $1^{\circ}$ de universidad (un grupo FC de control y otro FD que recibió enseñanza divergente) e investigadores en física.

En todos los casos (excepto en el grupo de investigadores) se verificó la homogeneidad estadística con resultados positivos significativos en conocimientos previos, producción divergente y nivel intelectivo. Se compararon los correspondientes niveles con dos pruebas de PD, DMU y DMS, hallando la diferencia de medias con la $t$ de student (nivel de significación $=0,05$ ).

El análisis de los resultados obtenidos nos permite resumir lo siguiente:

1) Si comparamos el grupo experimental $2 B$ de BUP se observa que existe una diferencia significativa a su favor respecto del grupo $2 \mathrm{~A}$ de control del mismo nivel.
2) El grupo experimental $2 \mathrm{~B}$ no muestra diferencias significativas en las citadas pruebas de PD respecto de los dos grupos de universidad ni tampoco respecto del grupo de investigadores de física.

3) El grupo de control 2A muestra resultados claramente inferiores respecto a todos los demás grupos excepto en DMU, con resultado prácticamente igual para dicho grupo y para los investigadores en física.

4) Si la comparación se realiza entre los restantes grupos de los demás niveles (universidad e investigadores en física), encontramos que en ningún caso se presentan diferencias significativas, ni entre los grupos de universidad ni entre éstos y los investigadores, excepto en la prueba DMU que es significativamente favorable para el grupo «convergente» FC de la universidad respecto del grupo de investigadores en física.

Dicho trabajo parece mostrar que la producción divergente y la creatividad científica en física, al menos en las pruebas utilizadas (DMU y DMS), no aumentan con la edad ni con los estudios o actividades científicas posteriores en dicha materia. No obstante, encontramos indicios de que los alumnos de menor edad son más susceptibles de experimentar cambios.

Nosotros quisimos aportar algo a dichos estudios. Para ello trabajamos durante el curso 1990-91 con las muestras ya mencionadas: dos grupos de BUP, 2A y 2D, y dos grupos de $\mathrm{COU}, \mathrm{CA}$ y $\mathrm{CB}$.

\section{METODOLOGÍA Y ESTRATEGIA EXPERI- MENTAL DE LA INVESTIGACION}

A. Muestras: Durante el curso 1990-91 trabajamos con 4 grupos de alumnos, dos de BUP y dos de COU, pertenecientes al IB «Las Veredillas», de Torrejón de Ardoz.

Dichos alumnos, de clase media, se repartían en los siguientes grupos:

- Grupos de BUP:

Grupo 2A: De $2^{\circ}$ de BUP, constaba de 28 alumnos, 19 mujeres y 9 varones, con una edad promedio de 15 años.

- Grupo 2D: De $2^{\circ}$ de BUP, constaba de 33 alumnos, 13 mujeres y 20 varones, con una edad promedio de 15 años.

- Grupos de COU:

Grupo CA: Constaba de 22 alumnos, 8 mujeres y 14 varones, con una edad promedio de 17 años.

- Grupo CB: Constaba de 15 alumnos, todos varones menos una mujer, con una edad promedio de de 17 años.

$B$. Contenidos teóricos impartidos: Están condicionados por el programa oficial, así como por las disposiciones del seminario de física del instituto. 
Se impartieron los temas establecidos para los grupos correspondientes en los que dominan los aspectos de mecánica y electricidad.

$C$. Tipo de enseñanza: Nuestra experiencia la llevamos a cabo en el caso de situaciones reales de aprendizaje. Tanto los grupos de BUP como los de COU recibieron una enseñanza tradicional, entendiendo por esto:

\section{C1. En clase:}

El alumno:

a) Asiste a la explicación del profesor.

b) Pregunta eventualmente alguna duda.

c) Realiza exámenes mensuales de problemas y cuestiones.

El profesor:

a) Explica los temas de física.

b) Responde a las preguntas que eventualmente le formulan los alumnos.

c) Elabora los exámenes procurando que las preguntas abarquen los distintos niveles de la taxonomía de Bloom (1975), lo cual incide en una predisposición en los alumnos más abierta y amplia al tener que enfrentarse con problemas o cuestiones que implican no sólo conocimiento o comprensión, como suele ser habitual, sino también aplicación, análisis, síntesis y evaluación.

\section{$C 2$. En el laboratorio:}

El profesor propone las prácticas a realizar.

El alumno las realiza siguiendo un guión y pregunta las dudas al profesor.

D. Variables psicológicas y académicas: Una vez elegidas las que, a nuestro juicio, tienen especial incidencia en el pensamiento en física para el presente trabajo, aprovechamos las pruebas o tests existentes en la literatura psicológica y, en caso de no existir, los adaptamos o los creamos. Tal ha sido el caso de nuestras pruebas de PD aplicadas a la física.

Enumeramos, a continuación, dichas variables, especificando las pruebas o tests usados para cuantificarlos:

- Inteligencia potencial, controlada por el test de las matrices progresivas de Raven (denotada R).

- Producción divergente en física, controlada por nuestras pruebas de PD (DMU, DMC, DMR y DMS) que mostramos en el anexo donde se explica cómo las evaluamos. Distinguimos las pruebas realizadas antes, al principio de curso, de las realizadas después, al final, añadiendo a las siglas las letras A (PDA) o D (PDD).

- Conocimientos previos en física, tanto de cuestiones convergentes como divergentes.

- Actitud hacia la física, controlada por la prueba «Diferencial Semántico», de Osgood (DS).
$E$. Tratamiento estadístico: Usamos fundamentalmente la diferencia de medias ( $t$ de student) y correlaciones (coeficiente de correlación de Pearson).

En el caso de la $t$ de student tomamos para $r$ un nivel de significación del 0,05.

En cuanto a los valores mínimos de $r$ significativos para el nivel de significación 0,05 para cada grupo son:
2A: 0,37
2D: 0,34
CA: 0,42
CB: 0,51

$F$. Homogeneidad: Para estudiar la homogeneidad de las muestras, consideramos, por una parte, a los grupos de BUP, 2A y 2D y, por otra, a los dos de COU, CA y CB:

- Para los grupos de BUP: Se comprueba la homogeneidad tanto en las pruebas de conocimientos previos como en el test de inteligencia general, actitud hacia la física y en el $92 \%$ de las variables de PD.

- Para los grupos de COU: se constata homogeneidad tanto en conocimientos previos como en inteligencia potencial, actitud hacia la física, así como en el $85 \%$ de las variables de PD.

\section{RESULTADOS, ANÁLISIS E INTERPRETA- CION}

Como ya hemos dicho, obtuvimos la diferencia de medias con la $t$ de student para analizar los cambios producidos y aplicamos el coeficiente de correlación de Pearson para determinar si se producían cambios de distribución interna en cada uno de los grupos anteriores.

Del análisis de los resultados para las pruebas de PD concluímos lo siguiente:

1) Si bien, al principio de curso, los dos grupos de BUP son homogéneos estadísticamente entre sí y también los dos grupos de COU, comparando BUP con COU (cuyos alumnos son dos años mayores que los de BUP), hay una moderada diferencia, del orden del $21 \%$, a favor de los de COU.

Dicha diferencia se produce más claramente en la prueba $\mathrm{DMR}$, en las variables originalidad $(\mathrm{O})$ y elaboración $(\mathrm{E})$.

2) Al comparar entre sí a los citados grupos de BUP y COU, al final del curso académico, dicha diferencia se redujo aproximadamente al $17 \%$.

Además, dicha diferencia ya no se da en la prueba de relaciones sino repartida entre las diferentes pruebas. Se da el caso de que uno de los grupos de BUP, el 2D, presenta ventaja sobre uno de los de COU en la variable originalidad $(\mathrm{O})$ de la prueba DMR.

3) En cuanto a la evolución de los grupos entre el principio del curso (octubre) y el final (mayo), se obser- 
Figura 2

Porcentaje en que progresan los alumnos de BUP (A, sector 1) y de COU (B, sector 1) en las pruebas de PD desde el principio hasta el final del curso.

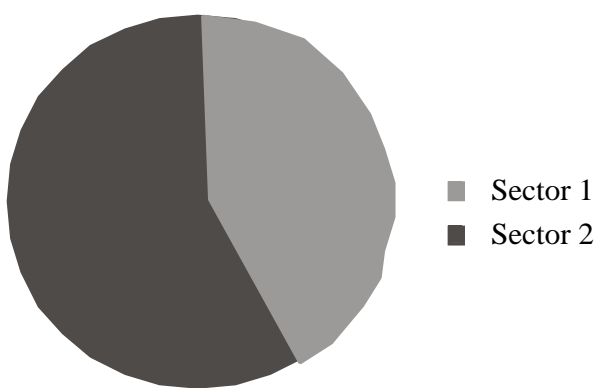

A

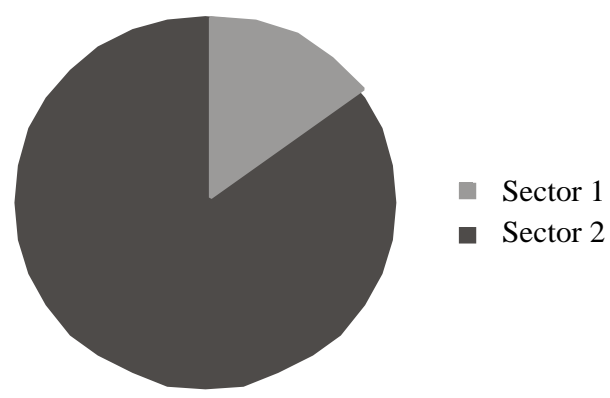

B va que, mientras los grupos de BUP progresan en un $42 \%$, los de COU lo hacen sólo en un $15 \%$ (Tablas I y II y figura 2).

Es de destacar específicamente:

- En DMR sólo evolucionan los grupos de BUP.

- En DMU evolucionan tanto los de BUP como los de COU.

- En DMC y en DMS no evolucionan ninguno salvo los grupos de BUP, que lo hacen ligeramente en la variable «originalidad» de la prueba DMS.

Como resumen de todo lo anterior estimamos que, al menos en nuestras pruebas de PD en física y en líneas generales, el pensamiento divergente cambia poco con la edad y la instrucción académica, observando, no obstante, un mayor cambio en los alumnos de menor edad, los de BUP, de 15 años.

En cuanto a nuestro estudio de la reestructuración de la PD, utilizando el coeficiente de correlación de Pearson, entre dichas pruebas para cada grupo antes y después del curso, vimos que el porcentaje de reestructuración es del $45 \%$ para el grupo $2 \mathrm{~A}$ y del $69 \%$ para el $2 \mathrm{D}$; en cuanto a los grupos de COU, resultan ser del $53 \%$ para el CA y del $96 \%$ para el CB.

Mientras parece normal que en los grupos de BUP haya más reestructuración que en los de COU, ya que BUP progresa más (en la prueba DMR sólo lo hace BUP y, efectivamente, se observa más reestructuración en los primeros), llama la atención que, en la prueba DMU, en la que ambos experimentan evolución -incluso más los de BUP- los de COU tienen una gran reestructuración y los de BUP apenas la experimentan.
Así, vemos que la evolución y la reestructuración, al menos en los períodos de edades estudiados, no tienen por qué experimentar un cambio paralelo.

El hecho de que, en la prueba de «unidades», ambos grupos evolucionen pero sea en los alumnos de COU en los que se dé una mayor reestructuración y en la de «relaciones» (que implica mecanismos más básicos, correspondientes a niveles superiores en la taxonomía de Bloom) sólo los alumnos de BUP experimenten tanto evolución como reestructuración nos hace pensar que podrían ser interesantes ulteriores investigaciones para estudiar, por ejemplo, si dichos mecanismos más básicos se establecen antes y los otros -más superficiales y susceptibles de cambio por la experiencia y conocimientos cotidianos- pueden originar reestructuración a intervalos más o menos largos de tiempo.

\section{ALGUNAS CONSIDERACIONES FINALES}

En nuestra civilización, la influencia de la física, de la ciencia en general y de la técnica es enorme. No obstante, para una gran mayoría de los ciudadanos lo es, fundamentalmente, por su utilidad práctica cotidiana. Hay desvinculación del ciudadano con el conocimiento científico y técnico.

El avance científico no ha ido acompañado de un desarrollo paralelo de su didáctica y metodología. Los conocimientos científicos han venido llegando con retraso al sistema educativo, cuya rigidez, además de incidir negativamente en la flexibilidad mental de profesores y alumnos, ha condicionando la creatividad y la capacidad innovadora del proceso de enseñanza-aprendizaje de la ciencia. 
Tabla I

Diferencia de medias entre las pruebas de PD antes y después del curso, PDA-PDD, para 2A y 2B. En la 1a. columna, 1a. casilla de cada fila, se indican las variables fluencia (F), flexibilidad (fx), originalidad (O) y elaboración (E) con los que se evalúan nuestras pruebas de PD (DMU, DMC, DMR y DMS). En las casillas con dos números, el superior es el valor medio y el inferior, la desviación típica. Entre ellas se da la diferencia de medias evaluada mediante la $t$ de student, indicando con el símbolo < un progreso para un nivel de significación de 0,05 .

\begin{tabular}{|c|c|c|c|c|c|c|}
\hline & $\begin{array}{c}\text { 2A } \\
\text { PDA }\end{array}$ & $\mathbf{t}$ & $\begin{array}{r}\text { 2A } \\
\text { PDD }\end{array}$ & $\begin{array}{c}\text { 2D } \\
\text { PDA }\end{array}$ & $\mathbf{t}$ & $\begin{array}{c}\text { 2D } \\
\text { PDD }\end{array}$ \\
\hline DMU.F & $\begin{array}{l}3,50 \\
1,55\end{array}$ & $\begin{array}{c}2,10 \\
<\end{array}$ & $\begin{array}{l}4,40 \\
1,62\end{array}$ & $\begin{array}{l}3,33 \\
1,85\end{array}$ & $\begin{array}{c}4,04 \\
<\end{array}$ & $\begin{array}{l}5,21 \\
2,10\end{array}$ \\
\hline$f x$ & $\begin{array}{l}1,18 \\
1,19\end{array}$ & $\begin{array}{c}3,11 \\
<\end{array}$ & $\begin{array}{l}2,32 \\
1,54\end{array}$ & $\begin{array}{l}1,03 \\
1,45\end{array}$ & $\begin{array}{c}3,92 \\
<\end{array}$ & $\begin{array}{l}2,73 \\
2,02\end{array}$ \\
\hline $\mathrm{O}$ & $\begin{array}{l}3,18 \\
2,13\end{array}$ & $\begin{array}{c}2,98 \\
<\end{array}$ & $\begin{array}{l}4,79 \\
1,91\end{array}$ & $\begin{array}{l}2,94 \\
2,11\end{array}$ & $\begin{array}{r}3,04 \\
<\end{array}$ & $\begin{array}{l}4,48 \\
1,99\end{array}$ \\
\hline DMC.F & $\begin{array}{l}4,04 \\
1,71\end{array}$ & 0,98 & $\begin{array}{l}4,54 \\
2,08\end{array}$ & $\begin{array}{l}3,85 \\
1,94\end{array}$ & 0,58 & $\begin{array}{l}3.61 \\
1.58\end{array}$ \\
\hline$f x$ & $\begin{array}{l}2,64 \\
1,47\end{array}$ & 0,86 & $\begin{array}{l}3,00 \\
1,63\end{array}$ & $\begin{array}{l}2,39 \\
1,54\end{array}$ & 0,16 & $\begin{array}{l}2,45 \\
1,51\end{array}$ \\
\hline $\mathrm{O}$ & $\begin{array}{l}4,57 \\
1,36\end{array}$ & 0,87 & $\begin{array}{l}4,91 \\
1,63\end{array}$ & $\begin{array}{l}4,32 \\
2,07\end{array}$ & 1,12 & $\begin{array}{l}4,81 \\
1,41\end{array}$ \\
\hline E & $\begin{array}{l}3,34 \\
1,39\end{array}$ & 0,91 & $\begin{array}{l}3,07 \\
0,80\end{array}$ & $\begin{array}{l}3,26 \\
1,85\end{array}$ & 0,25 & $\begin{array}{l}3,36 \\
1,43\end{array}$ \\
\hline DMR.O & $\begin{array}{l}2,21 \\
1,97\end{array}$ & $\begin{array}{c}3,40 \\
<\end{array}$ & $\begin{array}{l}4,33 \\
2,65\end{array}$ & $\begin{array}{l}2,66 \\
1,54\end{array}$ & $\begin{array}{c}4,68 \\
<\end{array}$ & $\begin{array}{l}4,75 \\
2,05\end{array}$ \\
\hline E & $\begin{array}{l}3,32 \\
1,63\end{array}$ & $\begin{array}{c}4,29 \\
<\end{array}$ & $\begin{array}{l}5,41 \\
1,99\end{array}$ & $\begin{array}{l}3,51 \\
1,68\end{array}$ & $\begin{array}{c}3,74 \\
<\end{array}$ & $\begin{array}{l}5,00 \\
1,54\end{array}$ \\
\hline DMS.F & $\begin{array}{l}3,36 \\
2,50\end{array}$ & 0,30 & $\begin{array}{l}3,18 \\
1,94\end{array}$ & $\begin{array}{l}2.15 \\
1.58\end{array}$ & 1,04 & $\begin{array}{l}2,54 \\
1,54\end{array}$ \\
\hline$f x$ & $\begin{array}{l}1,96 \\
1,89\end{array}$ & 0,33 & $\begin{array}{l}2,14 \\
2,05\end{array}$ & $\begin{array}{l}1,15 \\
1,54\end{array}$ & 0,98 & $\begin{array}{l}1,51 \\
1,48\end{array}$ \\
\hline $\mathrm{O}$ & $\begin{array}{l}3,21 \\
1,78\end{array}$ & 1,92 & $\begin{array}{l}4,16 \\
1,94\end{array}$ & $\begin{array}{l}3,14 \\
2,32\end{array}$ & $\begin{array}{c}2,17 \\
<\end{array}$ & $\begin{array}{l}4,34 \\
2,19\end{array}$ \\
\hline E & $\begin{array}{l}3,31 \\
1,86\end{array}$ & 1,41 & $\begin{array}{l}3,92 \\
1,37\end{array}$ & $\begin{array}{l}3,41 \\
1,81\end{array}$ & 1,48 & $\begin{array}{l}4,03 \\
1,56\end{array}$ \\
\hline
\end{tabular}


Tabla II

Diferencias de medias PDA-PDD para CA y CB. En la 1a. columna, 1a. casilla de cada fila, se indican las variables fluencia (F), flexibilidad $(\mathrm{fx})$, originalidad $(\mathrm{O})$ y elaboración (E) con las que se evalúan nuestras pruebas de PD (DMU, DMC, DMR y DMS). En las casillas con dos números, el superior es el valor medio y el inferior la desviación típica. Entre ellas se da la diferencia de medias evaluada mediante la

$t$ de student, indicando con el símbolo < un progreso para un nivel de significación de 0,05 .

\begin{tabular}{|c|c|c|c|c|c|c|}
\hline & $\begin{array}{c}\text { CA } \\
\text { PDA }\end{array}$ & $\mathbf{t}$ & $\begin{array}{c}\text { CA } \\
\text { PDD }\end{array}$ & $\begin{array}{c}\text { CB } \\
\text { PDA }\end{array}$ & $\mathbf{t}$ & $\begin{array}{c}\text { CB } \\
\text { PDD }\end{array}$ \\
\hline DMU. F & $\begin{array}{l}3,32 \\
1,64\end{array}$ & $\begin{array}{c}3,75 \\
<\end{array}$ & $\begin{array}{l}5,68 \\
2,46\end{array}$ & $\begin{array}{l}3,47 \\
1,55\end{array}$ & $\begin{array}{c}1,97 \\
<\end{array}$ & $\begin{array}{l}4,60 \\
1,59\end{array}$ \\
\hline$f x$ & $\begin{array}{l}1,36 \\
1,00\end{array}$ & $\begin{array}{c}4,85 \\
<\end{array}$ & $\begin{array}{l}3,27 \\
1,55\end{array}$ & $\begin{array}{l}1,27 \\
1,28\end{array}$ & $\begin{array}{c}2,27 \\
<\end{array}$ & $\begin{array}{l}2,47 \\
1,60\end{array}$ \\
\hline $\mathrm{O}$ & $\begin{array}{l}2,88 \\
1,36\end{array}$ & $\begin{array}{c}4,62 \\
<\end{array}$ & $\begin{array}{l}4,97 \\
1,63\end{array}$ & $\begin{array}{l}4,05 \\
2,16\end{array}$ & 0,93 & $\begin{array}{l}4,72 \\
1,74\end{array}$ \\
\hline DMC. F & $\begin{array}{l}3,86 \\
1,93\end{array}$ & 0,17 & $\begin{array}{l}3,95 \\
1,59\end{array}$ & $\begin{array}{l}4,40 \\
1,96\end{array}$ & 1,20 & $\begin{array}{l}5,07 \\
0,88\end{array}$ \\
\hline$f x$ & $\begin{array}{l}2,73 \\
1,73\end{array}$ & 0,00 & $\begin{array}{l}2,73 \\
1,52\end{array}$ & $\begin{array}{l}3,01 \\
1,79\end{array}$ & 1,43 & $\begin{array}{l}3,80 \\
0,86\end{array}$ \\
\hline $\mathrm{O}$ & $\begin{array}{l}5,18 \\
1,37\end{array}$ & 0,10 & $\begin{array}{l}5,13 \\
1,78\end{array}$ & $\begin{array}{l}5,87 \\
1,46\end{array}$ & 0,16 & $\begin{array}{l}5,95 \\
0,93\end{array}$ \\
\hline E & $\begin{array}{l}3,44 \\
1,69\end{array}$ & 0,31 & $\begin{array}{l}3,61 \\
1,94\end{array}$ & $\begin{array}{l}4,01 \\
2,27\end{array}$ & 0,15 & $\begin{array}{l}3,91 \\
1,15\end{array}$ \\
\hline DMR.O & $\begin{array}{l}2,37 \\
2,11\end{array}$ & 1,57 & $\begin{array}{l}3,44 \\
2,41\end{array}$ & $\begin{array}{l}4,65 \\
2,28\end{array}$ & 0,16 & $\begin{array}{l}4,81 \\
3,05\end{array}$ \\
\hline E & $\begin{array}{l}4,64 \\
1,87\end{array}$ & 0,37 & $\begin{array}{l}4,82 \\
1,37\end{array}$ & $\begin{array}{l}5,40 \\
2,06\end{array}$ & 0,67 & $\begin{array}{l}4,87 \\
2,26\end{array}$ \\
\hline DMS.F & $\begin{array}{l}3,45 \\
1.71\end{array}$ & 1,87 & $\begin{array}{l}4,59 \\
2,28\end{array}$ & $\begin{array}{l}2,80 \\
1,32\end{array}$ & 0,43 & $\begin{array}{l}3,00 \\
1,19\end{array}$ \\
\hline fx & $\begin{array}{l}2,36 \\
1,59\end{array}$ & 1,62 & $\begin{array}{l}3,18 \\
1,76\end{array}$ & $\begin{array}{l}1,67 \\
1,34\end{array}$ & 0,31 & $\begin{array}{l}1,80 \\
1,01\end{array}$ \\
\hline $\mathrm{O}$ & $\begin{array}{l}3,84 \\
1,99\end{array}$ & 0,54 & $\begin{array}{l}4,17 \\
2,17\end{array}$ & $\begin{array}{l}4,52 \\
2,15\end{array}$ & 0,55 & $\begin{array}{l}4,12 \\
1,82\end{array}$ \\
\hline E & $\begin{array}{l}3,39 \\
0,90\end{array}$ & 0,66 & $\begin{array}{l}3,59 \\
1,01\end{array}$ & $\begin{array}{l}3,72 \\
1,42\end{array}$ & 1,38 & $\begin{array}{l}4,39 \\
1,21\end{array}$ \\
\hline
\end{tabular}


Es importante que la investigación apoye un cambio que no consiste simplemente en cambiar contenidos sino en generar motivaciones y capacidades.

Así, el aprendizaje, obviamente, no ha de quedarse en el conocimiento de la información sino en utilizarla y relacionarla aplicándola a problemas nuevos o a situaciones inéditas. Pensemos que, habitualmente, el estudiante resuelve problemas preparados y bien estructurados, lo que, en general, no es el caso en la vida real, en la que surgen problemas que no se resuelven de forma reproductiva, pues no están estructurados, y ni siquiera están clarificadas las variables que intervienen y además no suelen tener una solución única, lo cual requiere precisamente los mecanismos mentales que llamamos producción o pensamiento divergente.

De ahí que nos parezca importante que se multipliquen las investigaciones en este campo donde nuestra metodología supone una nueva forma operativa de evaluación del PD y de la creatividad en el proceso de enseñanza-aprendizaje de la física, de las ciencias en general; y ofrece un marco de referencia en el que situar nuestras investigaciones aportando una estructura y elementos

\section{REFERENCIAS BIBLIOGRÁFICAS}

ABRA, J.(1989). Changes in creativity withage: Data, explanations and futher predictions. International Journal of Aging and Human Development, 28(2), pp. 105-106.

BLOOM, B.S. (1975). Taxonomy of Educational Objectives. Nueva York: David McKay Co.

GALTON, F. (1869). Hereditary genius: An inquiry into its laws and consequences. Londres: McMillan.

GUILFORD, J.P. (1967). The nature of human intelligence. Nueva York: McGraw Hill.

OWENS, A.J. (1973). Letter. Physics Today. Octubre, p. 9.

PASTOR SARRO, S. (1993). «Pensamiento divergente y creatividad científica en el proceso de enseñanza-aprendizaje de la física»,pp. 112-120. Universidad Autónoma de Madrid: Tesis doctoral.

PÉREZ FERNÁNDEZ, P. y CIVANTOS CARRILLO, M. (1985). «Estudio de la producción divergente en un curso de física a nivel de $2^{\circ}$ de BUP, utilizando el modelo de la estructura del intelecto de J.P. Guilford.» Primer Congreso Internacional Didáctica, Ciencias y Matemáticas. Barcelona. que suponen bases para un sistema organizativo científico a la manera de la estructura de las ciencias.

No vamos a sugerir posibilidades concretas de investigación relacionadas con nuestros trabajos; preferimos dejarlo a la imaginación del lector, pues creemos que las posibilidades son inmensas. Nos limitaremos a comentar que, en el marco del modelo EI de la estructura del intelecto de Guilford, hemos comprobado la especificidad del PD respecto de variables tanto psicológicas como académicas, el cual nos permite la investigación no sólo en el campo de la física sino de las demás ciencias. Así, en concreto, por ejemplo, hemos utilizado la taxonomía de Bloom (1975), que preconiza seis niveles jerárquicos en la actividad pensante, crecientes en complejidad y carácter divergente (conocimiento, comprensión, aplicación, análisis, síntesis y evaluación): los problemas de física pueden clasificarse en alguno de dichos niveles, con lo que podemos relacionar e investigar la inteligencia efectiva u operativa que se manifiesta al intentar resolver dichos problemas con su carácter divergente así como su correspondencia con las celdillas o actividades mentales del modelo de Guilford a través de nuestras pruebas de PD.
PÉREZ FERNÁNDEZ, P., CIVANTOS CARRILLO, M. y GASTESI A., M.R. (1987). «Estudio comparativo de producción divergente y estilo cognitivo en pruebas de PD en física en alumnos de $2^{\circ}$ de BUP, $1^{\circ}$ de Universidad e investigadores en física». II Congreso Internacional sobre investigación en la didáctica delas ciencias y de las matemáticas. ICE de la Universidad Autónoma de Barcelona-SFP de la Universidad de Valencia.

RIEBEN, L. (1978). Intelligence et pensèe créative. Neuchâtel: Delachaud-Niestlè. Trad. cast. (1974). Inteligencia global, inteligencia operatoria y creatividad. Barcelona: MédicaTécnica.

JENNIFER R. y SASSER-COEN, M.S. (1993): Qualitative Changes in Creativity in the Second Half of Life: A LifeSpand Developmental Perspective. Journal of Creative Behavior, 27(1), pp. 18-27.

STERNBERG, R.J. (1990). Wisdom: Its nature, origins and development. Cambridge: Cambridge University Press.

TSANG,T. (1974). Creativity versus age. Physics Today. Agosto, p. 9. 


\section{ANEXO}

\section{PRUEBAS DE PENSAMIENTO DIVERGENTE EN FÍSICA}

La calificación de dichas pruebas se realiza por la evaluación de las variables fluencia (F), flexibilidad (fx), elaboración (E) y originalidad (O). Para las tres primeras se realiza por el cómputo de las respuestas válidas y, para la originalidad, hay dos procedimientos, el primero de los cuales, el «estadístico», consiste en considerar una respuesta original cuando aparece un porcentaje de veces pequeño, acordado de antemano (por ejemplo un $2 \%$ ), y el segundo es el criterio del «evaluador» que utiliza las puntuaciones $0,1,2$ y 3 , según que la respuesta no tuviera ninguna originalidad, un poco, bastante o mucha originalidad, respectivamente.

He aquí las pruebas:

- Prueba DMU: Indica los usos múltiples que se te ocurran de un muelle de acero en física; por ejemplo, para calentarlo y estudiar la dilatación lineal de la pieza.

- Prueba DMC: ¿Qué fundamentos físicos, propiedades físicas, aplicaciones, principios físicos, etc., pueden encontrarse en común entre los componentes de este conjunto?
A Balanza
B Tijeras
C Rueda
D Péndulo
E Hoja de sierra, de acero
F Plomada
G Columpio
H Destornillador
J Barra de hierro
K Muelle de acero
L Pinzas
M Bola de acero
$\mathrm{N}$ Tabla de madera
O Martillo
$\mathrm{P}$ Vaso lleno de agua
Q Trozo de alambre
$\mathrm{R}$ Trozo de plástico
S Caja de zapatos
$\mathrm{T}$ Hoja de afeitar

Ejemplos:

ADGK: Pueden oscilar periódicamente.

DK: Pueden almacenar energía potencial.

BEHT: Ejercen mucha presión a causa de su filo.

- Prueba DMR: Cuando una barra metálica se calienta por uno de sus extremos, el calor se transmite a lo largo de ella hasta alcanzar el otro extremo. Imagina y describe un procedimiento experimental para estudiar este fenómeno, incluyendo las variables que pudiesen intervenir según tu criterio. 


\section{INVESTIGACIÓN DIDÁCTICA}

- Prueba DMS: Imagina y describe algunos experimentos de física que puedan ser realizados utilizando todos o algunos de los siguientes objetos:
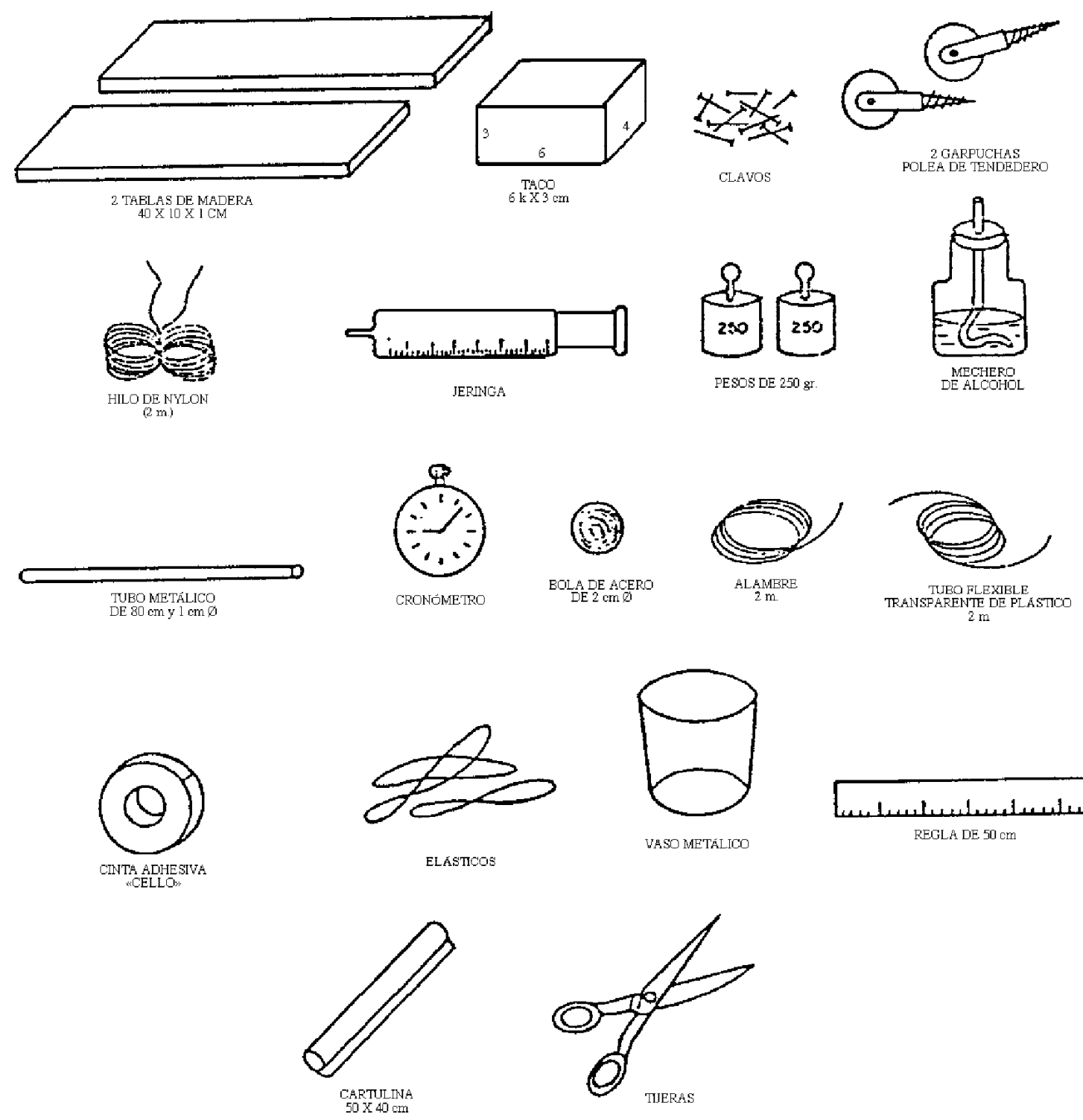